\title{
Mutual Interference Suppression Methods In Ultra-Wideband Navigation Systems With Combined Data Channel
}

\author{
Valery N. Bondarenko, \\ Timur V. Krasnov, \\ Vadim F. Garifullin \\ Engineering physics and radioelectronics institute, \\ Siberian Federal University, \\ Krasnoyarsk, Russian Federation \\ bers-redhold@ya.ru
}

\begin{abstract}
Effective mutual interference suppression methods are proposed for terrestrial ultra-wideband systems of mediumwave band. Navigation signals modulated with data provide higher accuracy, satellite and terrestrial systems integration. Such long range systems characterized by powerful mutual interferences.
\end{abstract}

Index Terms-Interference suppression, wideband navigation system, interference estimation, compensation.

\section{INTRODUCTION}

Terrestrial navigation systems characterized by high dynamic range of signals. Typically long range navigation systems (600 km and more) use longwave or mediumwave band and built on spread-spectrum signal propagation delay measuring. Followed by reducing the occupied bandwidth this navigation signals from base stations are divided by code. This signals are modulated with data bit to provide satellite and terrestrial systems integration.

At certain distance from the base station (about $50 \mathrm{~km}$ ) the cross-correlation function of powerful signal from a nearby station and low signal from a distant station prevents propagation delay measurement of the low signal. This powerful signal represents the mutual interference.

The mutual interference, also called adjacent-channel interference, is a spread-spectrum signal similar to useful signal but the amplitude, data bit, propagation delay, phase shift and frequency shift are different.

There are several ways to suppress mutual interference in the broadband system today, and one of effective methods is compensation. The compensator identifies interference from desired signal channel and subtracts interference copy from this channel.

Generally, after data bit of mutual interference is detected the interference copy is modulated with this bit. This method involves the input mixture delay equals interfering signal period. This delay is a serious restriction for device implementation $[1,2]$.
Due to promising precision and spectrum characteristics the signals considered in this paper have frequency modulation formats: MSK, MSK-BOC, SMSK, SMSK-BOC [1, 3].

\section{The Optimal Algorithm OF Propagation DELAy MEASUREMENT In PRESENCE OF Mutual INTERFERENCE AND NOISE}

The optimal measurement algorithm synthesis is derived for periodic spread-spectrum signal by using signal parameter estimation theory. Let the input mixture $y(t)$ is present on interval form 0 to $T s$, where $T s$ is spread-spectrum signal period:

$$
y(t)=s(t, \tau, \varphi, D)+s_{i}(t, \lambda)+n(t),
$$

where $s$ - is useful signal, $\tau$ and $\varphi$ - initial delay and phase (frequency is predetermined), $D$ - data bit, $s_{i}(t, \lambda)$ - mutual interference, $\lambda=(A, \mathrm{H}, \delta, F, \psi)$ - interference parameter vector, $\mathrm{H}$ - interference data bit, $A$ - amplitude, interference initial phase, $n(t)$ - additive white Gaussian noise with spectral density $N_{0} / 2$.

Using the observation from Eq. 1 and assuming parameters $\tau$ and $\varphi$ to be constant on signal period we obtain the likehood function equation:

$$
W(y(t) \mid \tau, \varphi, D, \lambda)=k \exp \left(\frac{2}{N_{0}} \int_{0}^{T s}\left[y(t)-s_{i}(t, \lambda)\right] s(t, \tau, \varphi, D) d t\right)
$$

where $k$ - the coefficient independent from $\tau, \varphi$ and $\lambda$.

After the known trigonometric conversions Eq. 2 is presented as 


$$
\begin{gathered}
W(y(t) \mid \tau, \varphi, \lambda)=k \exp \left(\frac{1}{N_{0}} Z(\tau) \cos (\varphi-\hat{\varphi})\right), \\
Z(\tau)=\sqrt{z_{1}^{2}(\tau)+z_{2}^{2}(\tau)} ; \hat{\varphi}=\operatorname{arctg}\left(z_{2}(\tau) / z_{1}(\tau)\right), \\
z_{1}(\tau)=\int_{0}^{T_{s}}\left[y(t)-s_{i}(t, \lambda)\right] s_{1}(t-\tau) d t ; z_{2}(\tau)=\int_{0}^{T s}\left[y(t)-s_{i}(t, \lambda)\right] s_{2}(t-\tau) d t ; \\
s_{1}(t)=I(t) \cos \left(\omega_{0} t\right)+Q(t) \sin \left(\omega_{0} t\right) ; \\
s_{2}(t)=-I(t) \sin \left(\omega_{0} t\right)+Q(t) \cos \left(\omega_{0} t\right),
\end{gathered}
$$

where $\omega_{0}-$ carrier frequency, $I(t)$ and $Q(t)$ - real and imaginary components of normalized complex envelope.

By averaging the conditional probability density in Eq. 3 through random phase with uniform distribution on $[-\pi ; \pi]$ the function $W$ transforms into:

$$
W(y(t) \mid \tau, \lambda)=\frac{1}{2 \pi} \int_{-\pi}^{\pi} W(y(t) \mid \tau, \varphi, \lambda) d \varphi=k I_{0}\left(\frac{1}{N_{0}} Z(\tau)\right),
$$

where $I_{0}(x)$ - the second order modified Bessel function. With given $I_{0}(x)$ monotony the optimal delay estimation algorithm according to maximum likehood criterion is presented as:

$$
\left.\max _{\tau} \ln W(y(t) \mid \tau)\right|_{\tau=\hat{\tau}}=\left.\max _{\tau} Z(\tau)\right|_{\tau=\hat{\tau}}
$$

where $\hat{\tau}$ is the maximum likehood evaluation of parameter $\tau$.

The optimal algorithm in Eq. 1 to 7 is obtained under the assumption that interference parameters are determined, so the algorithm cannot be implemented. As the practical importance, the algorithm determines potential achievable immunity applied to delay measurement. When interference parameters are not determined this algorithm needs to be supplemented by evaluation algorithm for parameter vector $\lambda$ (search procedure).

The optimal algorithm structure is presented on Fig. 1. The interference estimation block (IEB) produces optimal evaluation of interference parameters. The mixture of noise and interference is present on IEB input because the useful signal is subtracted by its copy formed by signal estimation block (SEB). The mixture of noise and the useful signal is present on SEB input because the interference is subtracted by its copy formed by the IEB. In case of powerful interference, the useful signal influence in the IEB is negligible. This fact allows to delete the useful signal subtractor from interference channel, so the compensation method becomes quasi-optimal.

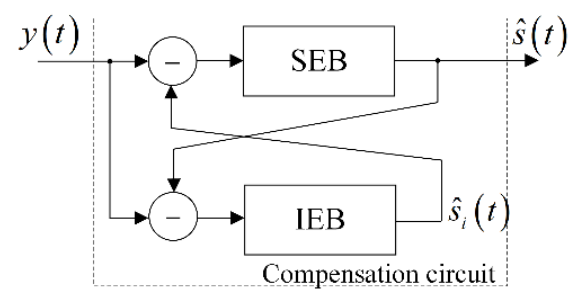

Fig. 1. Optimal interference compensation structure.

\section{DUAL-CHANNEL COMPENSATOR}

The dual-channel mutual interference compensator is shown on Fig. 2. The input mixture is present on inputs of two subtractors and interference estimation block, that consists of code and phase synchronization circuits, amplitude evaluation circuit and quadrature modulator. In order to provide high precision tracking for interference parameters the circuits contain discriminators that are optimal for periodic MSK-, MSK-BOC, SMSK- or SMSK-BOC signals (see Eq. 5) [4]. Delay and phase filtering is provided by the Type 2 control systems. Amplitude evaluation circuit is presented as the inphase channel of phase discriminator, completed with signfunction on the output of integrator for $z_{1}$ (see Eq. 5). Sign is evaluated at the end of interference period $(T S)$.

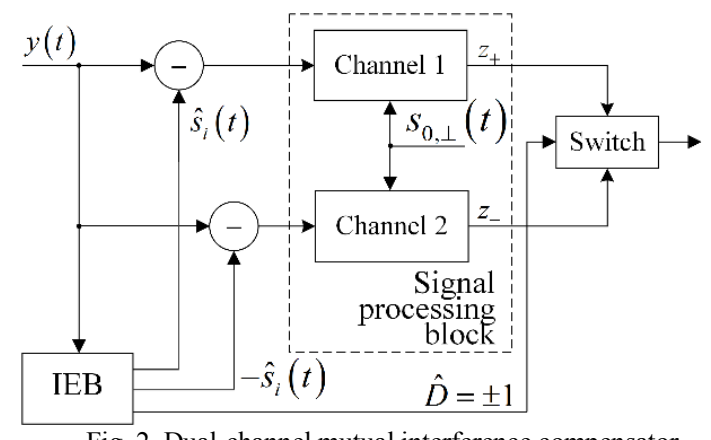

Fig. 2. Dual-channel mutual interference compensator.

Interference copy $\hat{s}_{i}=s_{i}(\hat{\tau}, \hat{\varphi}, \hat{F})$ of normalized amplitude is multiplied by amplitude evaluated in the amplitude evaluation unit, then it's completed with data bit $D=1$. Another interference copy is completed with data bit $D=-1$. Both the copies are fed to respective subtractors. After subtractors the mixture is present at signal processing block.

The useful signal processing block is the two identical correlation channels. On the one input the mixture is free from interference modulated with $D=1$, and on another input the mixture is free from interference with the opposite data bit. The mixture is processed at each of two correlation channels: the correlation function of the mixture and the reference signal is produced. After the end of interference period its data bit is detected accurately and the controls the switch (see Fig. 2). Thus the switch provides correctly processed mixture to the receiver output.

This scheme advantage is the useful signal processing with suppressed interference independently from interference data bit. This scheme implementation requires doubling hardware resources significantly increasing equipment cost and size. 
IV. COMPENSATOR WITH INPUT SIGNAL BLANKING

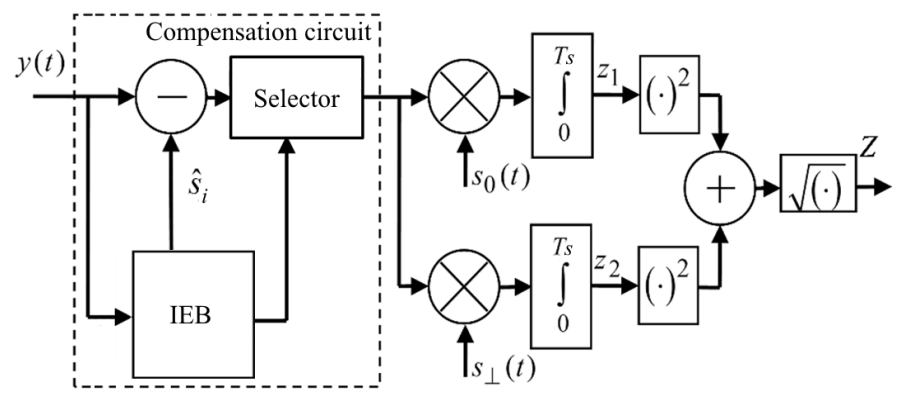

Fig. 3. Compensator with input signal blanking

Input signal blanking compensator circuit is presented on Fig. 3. The interference estimation block, described above, evaluates interference parameters including the data bit. During the $T s$ period the $z_{1}$ value (see Eq. 5) in IEB is rising monotonically due to high power of interference. Sign of integrator output value is being sampled at every pseudorandom noise sequence (PRS) symbol period. This function represents the interference data bit evaluation. Then after a few symbols the data bit evaluation has enough reliability to be taken into interference copy forming. Until data bit is evaluated the selector (see Fig. 3) blanks input of the useful signal receiver. The data bit is evaluated for the first time after the first PRS symbol, and then it's specified at each following symbol. When $z_{1}$ exceeds the predetermined threshold the IEB switches selector into the pass mode. Then the input signal with suppressed interference is being processed in the useful signal correlation receiver. After interference period the selector is being switched into cancellation mode.

This circuit advantage is the impulse noise neutralization. The impulse noise will occur if interference copy with undetected data bit is subtracted from the input, thus the interference either is reduced or twice increased. This method provides the useful signal power loss, but this loss is negligible with large PRS length.

\section{RESULTS}

At high range ultra-wideband navigation systems applications, the most effective mutual interference suppression method is input signal blanking compensator built on optimal discriminators, Type 2 control systems and realtime data bit evaluation circuit. During the data bit detecting the receiver input is cancelled preventing impulse noise entry. First PRS symbol blanking provides significant reducing of negative effect related with random data bit of the interference.

For $600 \mathrm{~km}$ systems range with $80 \mathrm{~dB}$ dynamic range the PRS length may be assumed as 16383 symbols. Thus the useful signal power loss is negligible.

Another disadvantage is cross-correlation properties degradation: autocorrelation function of non-modulated signals may reduce on $6 \mathrm{~dB}$ when blanking one of 16383 PRS symbols.

\section{ACKNOWLEDGMENT}

The report study was funded by RFBR according to the research projects № 16-38-00171, № 16-48-243024.

\section{REFERENCES}

[1] A. A. Gorchakovskiy, V. V. Evstratko, A. V. Mishurov, S. P. Panko, S. A. Ryabushkin, V. A. Shatrov, and V. V. Sukhotin, "Some Design Aspects of Command and Control Systems for Spacecrafts," 2013 International Siberian Conference on Control and Communications (SIBCON), Proceedings, Siberian Federal University, Krasnoyarsk, Russia, 12-13 September, 2013. [IEEE Catalog Number: CFP13794-CDR. ISBN: 978-1-4799-1060-1] DOI: 10.1109/SIBCON.2013.6693649

[2] Ye. A. Litinskaya, V. S. Panko, S. V. Polenga, and Yu. P. Salomatov, "Low profile phased array antenna with combined electronical and mechanical beam steering for satellite communications," 24nd Int. Crimean Conference "Microwave \& Telecommunication Technology" (CriMiCo'2014), Sevastopol, Crimea, Russia, pp. 461-462, 7-13 September 2014, DOI: 10.1109/CRMICO.2014.6959479

[3] V. N. Bondarenko, R. G. Galeev, and A. G. Klevlin, "Perspective Ways of Modulation in Broadband Radio Navigating Systems," Journal of Siberian Federal University. Engineering \& Technologies, 4th ed., vol. 1, Krasnoyarsk, Russia: Siberian Federal University, 2011, pp. 17-24.

[4] V. N. Bondarenko and V. I. Kokorin, "Wideband navigation systems with frequency shift keying spread-spectrum signals", Novosibirsk, Russia: Nauka, 2011. 